\title{
Human mesenchymal stem cells promote CD34phematopoietic stemcell proliferation with preserved red blood cell differentiation capacity
}

\begin{abstract}
Studies showed that co-transplantation of mesenchymal stem cells (MSCs) and cord bloodderived $\mathrm{CD}_{3} 4^{+}$hematopoietic stem cells (HSCs) offered greater therapeutic effects but little is known regarding the effects of human Wharton's jelly derived MSCs on HSC expansion and red blood cell (RBC) generation in vitro. This study aimed to investigate the effects of MSCs on HSC expansion and differentiation. HSCs were co-cultured with MSCs or with 10\% MSCs-derived conditioned medium, with HSCs cultured under standard medium served as a control. Cell expansion rates, number of mononuclear cell post-expansion and number of enucleated cells post-differentiation were evaluated. HSCs showed superior proliferation in the presence of MSC with mean expansion rate of $3.5 \times 10^{8} \pm 1.8 \times 10^{7}$ after day 7 compared to the conditioned medium and the control group $\left(8.9 \times 10^{7} \pm 1.1 \times 10^{8}\right.$ and $7.0 \times 10^{7} \pm 3.3 \times 10^{6}$ respectively, $\left.\mathrm{P}<0.001\right)$. Although no significant differences in $\mathrm{RBC}$ differentiation were observed between groups at passage IV, the number of enucleated cell was greater compared to earlier passages, indicating successful RBC differentiation. Cord blood-derived CD34 ${ }^{+}$HSCs can be greatly expanded by co-culturing with MSCs without affecting the RBC differentiation capability, suggesting the importance of direct MSC-HSCs contact in HSC expansion and RBC differentiation.
\end{abstract}

Keyword: Hematopoietic stem cells; Mesenchymal stem cells; Red cell differentiation 